\title{
Daños por depredación y tamaño de concha del caracol diádromo Neritina virginea (Gastropoda: Neritidae) en el Río Mameyes, Puerto Rico
}

\author{
Juan Felipe Blanco-Libreros ${ }^{1} \&$ Andrea Arroyave-Rincón ${ }^{2}$ \\ 1. Instituto de Biología, Facultad de Ciencias Exactas y Naturales, Universidad de Antioquia, Medellín Colombia. \\ Apartado Aéreo 1226; blanco@exactas.udea.edu.co \\ 2. anar01@matematicas.udea.edu.co
}

Recibido 20-XI-2008. Corregido 22-II-2009. Aceptado 26-III-2009.

\begin{abstract}
Predator damage and shell size on the diadromous snail Neritina virginea (Gastropoda: Neritidae) in the Mameyes River, Puerto Rico. We compared predators' damage with shell size in live individuals and empty shells $(\mathrm{n}=5066)$ of the snail Neritina virginea in the Mameyes River (Puerto Rico, Greater Antilles). According to the literature and direct observations, damages on empty shells were attributed to predation by aquatic birds (e.g. Gallinula chloropus) and decapods (e.g. Macrobrachium spp.), while damages on live individuals were due to rasping by co-specifics and erosion. Predation by decapods and birds, as estimated by the proportion of empty shells, was low ( 2 and $0.36 \%$, respectively). Shell size was significantly different between types of predators (range: decapods: $3.5-15.0 \mathrm{~mm}$, birds: $8.1-19.4 \mathrm{~mm}$ ). By comparing sizes of the empty shells and the live individuals, we concluded that decapods specialize on large groups of small migratory juveniles, while birds specialize on the largest resident individuals. Worn shells were highly frequent in both empty shells and live individuals, and sizes did not differ between samples. A comparison by slow-flow and fast-flow habitats showed that predators do not discriminate shell sizes between environments. However, the frequency of damage by birds and decapods was greater under slow-flow conditions. Despite of the little contribution of predation to the population dynamics in this species, predation might be an important driver of size-dependent behavioral responses such as upstream migration and microhabitat selection. Rev. Biol. Trop. 57 (4): 1069-1080. Epub 2009 December 01.
\end{abstract}

Key words: diadromous snails, size-dependent effects, shell damage, predation.

Una característica sobresaliente de varios géneros de gasterópodos de agua dulce de la familia Neritidae (Gastropoda) es que sus juveniles migran estacionalmente río arriba en agrupaciones masivas (Schneider \& Lyons 1993, Pyron \& Covich 2003, Blanco \& Scatena 2005) debido a su ciclo de vida diádromo obligado (Crandall 1999). A medida que los individuos crecen este comportamiento migratorio reofilico se reduce y los individuos empiezan a establecerse a lo largo del río (Schneider \& Lyons 1993, Blanco \& Scatena 2006, 2007). De esta forma, las agregaciones migratorias en las zonas bajas de los ríos se componen principalmente de los individuos más pequeños, mientras que en las zonas altas se componen de individuos más grandes pero en menor densidad (Schneider \& Lyons 1993). Como consecuencia el tamaño promedio de los individuos de las poblaciones aumenta con la distancia desde la desembocadura (Pyron \& Covich 2003, Blanco 2005).

Schneider \& Lyons (1993) propusieron que la migración masiva río arriba en juveniles del gasterópodo nerítido Neritina latissima de la costa Pacífica de Costa Rica era impulsada por un alto riesgo de depredación en el estuario y en la parte baja del río impuesto por peces 
moluscívoros. En experimentos y observaciones de campo, ellos observaron que los juveniles se escondían bajo las rocas ante la presencia de peces depredadores como Sphoeroides annulatus, mientras que los individuos grandes permanecían expuestos.

De acuerdo a su modelo conceptual, los individuos más grandes tienen menos riesgo de ser depredados porque alcanzan un tamaño que les confiere "refugio" (ver hipótesis del tamaño eficiente; Lampert \& Sommer 1997) y se espera que permanezcan en un lugar determinado. Los gasterópodos de tamaños pequeños e intermedios tienen una mayor susceptibilidad $\mathrm{y}$, debido posiblemente a menores costos energéticos para moverse en contra de la corriente, se espera que se desplacen continuamente río arriba. Estos gasterópodos disminuirían su riesgo de depredación, alcanzarían una mayor sobrevivencia y fecundidad moviéndose una distancia intermedia, siempre y cuando minimicen el costo energético y el tiempo invertido en la migración.

Se conoce ampliamente que los gasterópodos nerítidos de agua dulce son parte importante de la dieta de peces, en particular de los Eleotridae y Tetraodontidae que habitan los estuarios y partes bajas de los ríos insulares y costeros (Puerto Rico: Warmke \& Erdman 1963, Corujo-Flórez 1980, Costa Rica: Alpírez et al. 1984, Brazil: Texeira 1994, Polinesia Francesa: Resh et al. 1999, Bahamas: Layman \& Silliman 2002). Sin embargo, existen pocos estudios sobre otros depredadores, aunque se conoce que aves y decápodos incluyen nerítidos en sus dietas (Ford 1979, Pyron \& Covich 2003). Contrariamente a la depredación por peces, los cuales engullen a sus presas, la depredación por aves y decápodos deja marcas conspicuas en las conchas que permiten establecer su identidad (Vermeij 1993). Adicionalmente, dichos depredadores pueden ser altamente selectivos en cuanto a los tamaños utilizados debido a una relación inversa entre el beneficio de utilizar presas más grandes y el costo de manipulación (Vermeij 1993).

La depredación sobre Neritina virginea ha sido abordada de manera anecdótica (e.g.
Pyron \& Covich 2003) y solamente se conoce que hace parte de la dieta de peces gracias a estudios de contenidos estomacales (e.g. Corujo-Flórez 1980, Texeira 1994, Layman \& Silliman 2002). Publicaciones recientes han mencionado que los individuos son significativamente más grandes pero menos densos en condiciones de flujo menos turbulento, mientras que los individuos son significativamente más pequeños pero más densos en flujos menos turbulentos (Blanco \& Scatena 2005, 2007). Adicionalmente, se ha demostrado que durante las migraciones río arriba, los juveniles prefieren la última condición, lo que sugiere que la distribución espacial de los individuos entre y dentro de hábitats, y que la selección de las rutas de migración, responde al riesgo de depredación relacionado con la naturaleza hidráulica de los cauces (Blanco \& Scatena 2007).

Este artículo busca establecer: 1) los tipos de daños de las conchas, principalmente por depredación, 2) las distribuciones de tamaños de las conchas entre tipos de daños, 3) si el tamaño de conchas seleccionadas por un tipo particular de depredador corresponden con las de los individuos juveniles que exhiben comportamiento migratorio río arriba, y 4) si existen diferencias de frecuencia de depredación y selección de tamaños de conchas entre hábitats contrastantes en términos de turbulencia del flujo de agua.

\section{MATERIALES Y MÉTODOS}

Especie estudiada: El gasterópodo Neritina virginea (L.) se encuentra desde la Península de la Florida y las islas Bahamas hasta el norte de Brazil, y desde el Golfo de México y América Central hasta las Antillas (Blanco 2005). El tamaño oscila entre 4 y $25 \mathrm{~mm}$, desde los individuos recientemente reclutados hasta los adultos más grandes. Los juveniles y adultos exhiben una coloración verde con un patrón variable de líneas axiales o de pequeñas pinceladas amarillas (Blanco \& Scatena 2005). Habitan en una forma marina, que invade la vegetación sumergida de las 
lagunas costeras (García-Cubas 1981) y las raíces aéreas y neumatóforos de los manglares (J.F. Blanco observaciones personales), pero también se encuentran densas poblaciones sobre las piedras desde la desembocadura de los ríos hasta varios kilómetros aguas arriba (Pyron \& Covich 2003, Blanco \& Scatena 2006).

Área de estudio y muestreo: Este trabajo se realizó entre Agosto 22 de 2000 y Septiembre 31 de 2003 en el Río Mameyes, Noreste de Puerto Rico, cerca al límite inferior de la Reserva Forestal El Yunque (antes llamada Bosque Nacional del Caribe) administrada por el Servicio Forestal de los Estados Unidos para proteger las partes media y superior de la cuenca de varios ríos que drenan la Sierra de Luquillo. El sitio de estudio se ubica debajo de puente 1771 de la carretera PR $3\left(18^{\circ} 22^{\prime} 27^{\prime \prime} \mathrm{N}\right.$ y $65^{\circ} 45^{\prime} 50^{\prime}$ ' W, altitud: $5 \mathrm{~m}$ sobre el nivel del mar) sobre el Río Mameyes. El sitio de estudio está ubicado $2 \mathrm{~km}$ aguas arriba de la desembocadura y $1.3 \mathrm{~km}$ desde el límite superior del estuario y fue descrito en detalle en Blanco \& Scatena (2005, 2006, 2007).

La presencia del pilote que soporta el puente ha dividido el lecho del río en dos cauces húmedos (principal y secundario) paralelos que se unen 50m aguas abajo. En el cauce principal el agua fluye de manera más turbulenta que en el cauce secundario, lo que promueve una mayor profundidad en el primero que en el segundo (Cuadro 1). Se ha publicado anteriormente (Blanco \& Scatena 2005, 2007) que los individuos son significativamente más grandes en el cauce secundario que en el principal, y que durante las migraciones río arriba los juveniles prefieren este último, lo que sugiere que este comportamiento responde al riesgo de depredación relacionado con la naturaleza hidráulica de los cauces.

En este trabajo utilizamos datos parciales (tamaños de individuos vivos) publicados por Blanco \& Scatena $(2005,2007)$ y datos inéditos de conchas vacías colectados durante dichos estudios. Para establecer la distribución de tallas de los individuos (vivos) de la población, se realizó el muestreo cuando el caudal del río fue menor que $10 \mathrm{~m}^{3} / \mathrm{s}$ en los dos cauces del Río Mameyes bajo el puente de la carretera PR3. Durante el primer año el muestreo fue semanal (Blanco \& Scatena 2005, 2007) y posteriormente mensual (Blanco 2005). Los individuos fueron colectados al azar dentro de diez $(n=10)$ cuadrados de PVC de $0.5 \times 0.5 \mathrm{~m}$ puestos en el lecho del río en ambos cauces. El ancho de las conchas fue medida con un calibrador como un estimativo de la talla (Blanco \& Scatena 2005). De acuerdo al tamaño los individuos se pueden agrupar como

\section{CUADRO 1}

Características de los dos cauces estudiados en la parte baja del rio Mameyes: CP: Cauce principal, CS: Cauce secundario. Se indican los valores promedios y el ámbito (entre paréntesis) medidos durante caudal bajo $\left(1 \mathrm{~m}^{3} / \mathrm{s}\right)$. Datos tomados de Blanco y Scatena $(2005,2007)$

TABLE 1

Characteristics of the two study reaches in lower Rio Mameyes. CP: Main reach, CS: Side reach. Mean and range (within parentheses) measured at low discharge $\left(1 \mathrm{~m}^{3} / \mathrm{s}\right)$. Modified from Blanco y Scatena $(2005,2007)$

$\begin{array}{lcc}\text { Variable } & \text { CP } & \text { CS } \\ \text { Ancho de cauce }(\mathrm{m}) & 11 & 3 \\ \text { Profundidad }(\mathrm{cm}) & 20(5-30) & 30(10-50) \\ \text { Velocidad de fondo }(\mathrm{m} / \mathrm{s}) & 0.15(0.00-1.00) & 0.20(0.00-0.50) \\ \text { Número de Reynolds } & 3000(0-20000) & 4000(0-10000) \\ \text { Substratos dominantes } & \text { Bloques y cantos } & \text { Cantos y cascajo } \\ \text { Densidad de } N \text {. virginea }(\text { ind./m² } & 116(0-800) & 17(0-160) \\ \text { Tamaño de } N \text {. virginea }(\mathrm{mm}) & 6.2(2.2-18.5) & 7.6(4.4-17.0)\end{array}$


reclutas $(<4 \mathrm{~mm})$, juveniles $(4-8 \mathrm{~mm})$ y adultos ( $>8 \mathrm{~mm}$ ) (Blanco \& Scatena 2005). Centenares de reclutas y juveniles se agregan durante la época de lluvias para migrar río arriba después de cada evento de creciente pero pocos adultos se integran a estos grupos (Blanco \& Scatena 2005, 2007). Para establecer la distribución de tallas de los individuos que exhibían comportamiento migratorio, éstos se colectaron al azar dentro de grupos migratorios que cubrían áreas de hasta $21 \mathrm{~m}^{2}$ sobre el lecho del río o que formaban hileras de hasta varios metros durante siete fechas diferentes entre agosto de 2000 y junio de 2001 (Blanco \& Scatena 2005). Se colectaron un total de 4786 individuos tanto de población establecida en general (POP TOTAL 3809) como de los juveniles migratorios (MIGRANTE=977).

Durante el mismo periodo de estudio y dentro de los mismos cuadros en que se recolectaron los individuos vivos, se recolectaron conchas vacías con diferentes daños. Estas se clasificaron en las siguientes categorías de daños antes de ser medidas: conchas sin daño o intactas (SD), conchas rotas (R), conchas con desgaste (DG), conchas depredadas por ave pero sin desgaste (DPA), conchas depredadas por ave y con desgaste (DPA+DG), conchas depredadas por decápodos pero sin desgaste (DPD) y conchas depredadas por decápodos y con desgaste (DPD+DG). Las conchas depredadas por ave presentan una perforación dorsal en la voluta del cuerpo en el lugar en donde está sujeto el músculo de la columnela. Las depredadas por decápodos presentan dos perforaciones laterales, una en el hombro cerca a la espira y otra cerca al callo, dejadas por la acción de la quela con la cual camarones o cangrejos sujetan al gasterópodo (Fig. 1). La otra quela es utilizada para retirar el opérculo y extraer el cuerpo a través de la abertura (Vermeij 1993). En el sitio de estudio fue frecuentemente observada la polla de agua Gallinula chloropus y algunos individuos

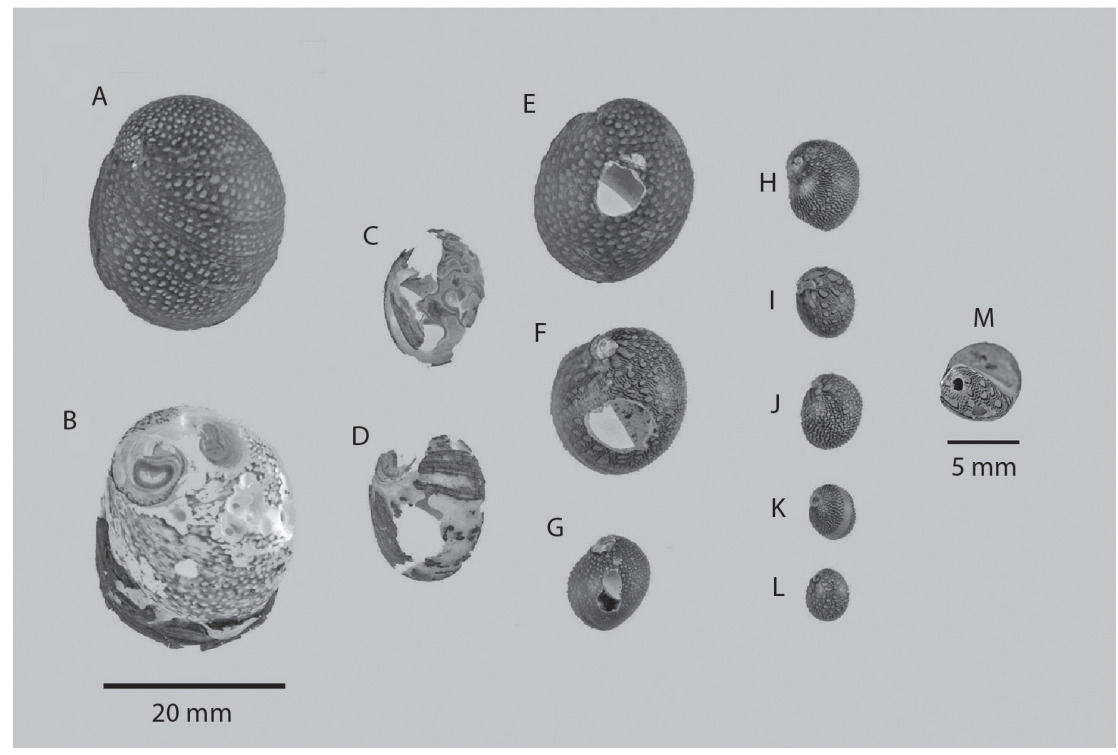

Fig. 1. Tipos de daños observados en las conchas de N. virginea. A. Conchas intactas. B. Daño por desgaste. C-D. Conchas rotas. E-G. Conchas depredadas por aves (nótese la perforación dorsal). H-L. Conchas depredadas por decápodos (las perforaciones laterales no se observan). M. Vista ventral y ampliada de la concha I en la que se observan las perforaciones realizadas por la quela de un decápodo.

Fig. 1. Damages observed on shells of N. virginea. A. Intact shells. B. Eroded shells. C-D. Broken shells. E-G. Shells preyed by birds (note dorsal perforation). H-L. Shells preyed by decapods. M. Ventral-magnified view of the shell shown in I. Note piercings made by decapod claws. 
pequeños del camarón Macrobrachium spp. Pyron \& Covich (2003) observaron cangrejos de agua dulce (Epilobocera sinuatifrons) "cargando conchas" en la parte alta de este mismo río. Sin embargo, durante nuestro estudio no se observaron cangrejos, ni tampoco cayeron en trampas en faenas recientes. Finalmente, el desgaste de las conchas ocurre por el raspado que se causan los individuos de $N$. virginea unos a otros supuestamente para suplementarse con carbonato de calcio, el cual se encuentra en menor concentración que en el mar (Ferguson 1959). Un número variado de orificios superficiales son dejados en las conchas y en algunos casos todo el periostraco es removido llegando inclusive a perforar completamente la voluta del cuerpo (Fig. 1).

Aunque también se realizaron censos visuales subacuáticos de depredadores en cada uno de los cauces, la abundancia por cuadro fue tan baja que sólo se consideró la presencia o ausencia para cada cauce a través de las fechas de muestreo.

Análisis de datos: Para un primer análisis, los tamaños de las conchas fueron transformados logarítmicamente y se compararon entre categorías utilizando un Análisis de Varianza de Una Vía (ANOVA) en el programa estadístico Statgraphics Plus versión 5.1. Adicionalmente, se calculó el porcentaje de individuos en cada categoría de daño para estimar el impacto de la depredación. No se hizo un análisis temporal debido a la baja abundancia de conchas vacías (280) y a la alta variabilidad entre una fecha y otra (abundancias mínimas y máximas: 1-26).

Para un segundo análisis, se consideraron las siguientes categorías: DPA, DPD, R y DG. Las conchas que presentaron combinaciones de marcas de depredación y desgaste fueron incluidas bajo la primera categoría para aumentar el tamaño de muestra y realizar comparaciones entre hábitats. Se utilizó un Análisis de Varianza de dos vías para evaluar los efectos independientes del hábitat y tipo de daño y la interacción entre las dos fuentes de variación. Finalmente, se realizó un Análisis Cruzado de Chi-Cuadrado para comparar las abundancias entre tipos de daño y hábitats.

\section{RESULTADOS}

Del total $(n=5066)$ de individuos recolectados, vivos y muertos, se estimó que el 5.53\% son individuos muertos. De éstos el $36.43 \%$ fueron aparentemente depredados por crustáceos y el $6.43 \%$ por aves, ya sea que las conchas presentaran desgaste o no. Del $36.43 \%$ de conchas depredadas por decápodos el $23.93 \%$ de las conchas presentó desgaste y del $6.43 \%$ depredadas por aves el $4.64 \%$ reveló este tipo de daño. El 18\% de las conchas vacías estaban rotas y solo el $2.8 \%$ intactas.

Se observaron diferencias significativas de los tamaños de las conchas entre los diferentes tipos de daños (Cuadro 2). Los tamaños de

CUADRO 2

Análisis de varianza de una vía comparando el tamaño de los individuos vivos y de las conchas vacías de Neritina virginea transformados logaritmicamente

TABLE 2

One-Way analysis of variance comparing log-10 transformed sizes of live individuals and empty shells of Neritina virginea

\begin{tabular}{|c|c|c|c|c|c|}
\hline $\mathrm{FV}^{*}$ & $\mathrm{SC}^{*}$ & GL* & $\mathrm{CM}^{*}$ & VF* & $\mathrm{Pb}^{*}$ \\
\hline Entre grupos & 13.37 & 8 & 1.67 & 76.70 & $<0.0001$ \\
\hline Error & 110.17 & 5057 & 0.02 & & \\
\hline Total Corregido & 123.54 & 5065 & & & \\
\hline
\end{tabular}


las conchas vacías encontradas con desgaste variaron entre 3.2 y $22.8 \mathrm{~mm}$, las rotas variaron entre 4.4 y $21.8 \mathrm{~mm}$ y las intactas entre 2.9 y $21.2 \mathrm{~mm}$ (Fig. 2) y no fueron estadísticamente diferentes. Las conchas depredadas por decápodos variaron entre 3.5 y $15.0 \mathrm{~mm}$ y no presentaron diferencias significativas ya fuesen intactas o desgastadas. Igualmente, no presentaron diferencias significativas con la muestra de individuos migrantes (ámbito: 2.4-12.9mm). Las conchas depredadas por aves variaron entre 8.1 y $19.5 \mathrm{~mm}$ y tampoco presentaron diferencias significativas de tallas, tanto en estado intacto o desgastado. El desgaste, sin embargo, afectó los límites superior e inferior de tallas de las conchas depredadas por camarones y aves, respectivamente. Finalmente, las conchas depredadas por aves fueron significativamente más grandes que las depredadas por decápodos, y correspondieron al $60 \%$ superior $(>8 \mathrm{~mm})$ de la distribución de tallas de los individuos de toda la población establecida (ámbito: 2.1$21.4 \mathrm{~mm})$.

La comparación de conchas desgastadas, rotas y depredadas entre los dos hábitats mostró los siguientes resultados. Las conchas depredadas por decápodos fueron significativamente más abundantes que las depredadas por aves, independientemente del hábitat (39.9\% y 9.6\%, respectivamente; $\chi^{2}=16.54$, G.L. $=3, \mathrm{P}<0.001$; Fig. 3). El cauce secundario presentó una mayor proporción tanto de conchas depredadas por aves como por decápodos, pero la diferencia fue mayor en las primeras. Las conchas desgastadas y rotas también fueron más abundantes en el cauce secundario.

El tamaño de las conchas vacías no mostró diferencias significativas entre hábitats, pero si entre tipos de daños (Cuadro 3). Tampoco mostró una interacción significativa entre las



Fig. 2. Comparación entre los tamaños (datos sin transformar) de las características de conchas presentes en los individuos vivos y conchas vacías de Neritina virginea. POP TOTAL: conchas de la población total de individuos vivos; MIGRANTES: conchas de juveniles migrantes vivos; DPD: conchas vacías depredadas por decápodo; DPD+DG: conchas vacías depredadas por decápodo y con desgaste; DPA: conchas vacías depredadas por ave; DPA+DG: conchas vacías depredadas por ave y con desgaste; DG: conchas vacías desgastadas; R: conchas vacías rotas; SD: conchas vacías intactas.

Fig. 2. Comparison of untransformed sizes among shell characteristics in live individuals and empty shells of Neritina virginea. POP TOTAL: population live established individuals; MIGRANTES: migratory live juveniles; DPD: empty shells preyed by decapods; DPD+DG: empty shells preyed by decapods and eroded; DPA: empty shells preyed by birds; DPA+DG: empty shells preyed by birds and eroded; DG: empty eroded shells; R: empty broken shells; SD: empty intact shells. 


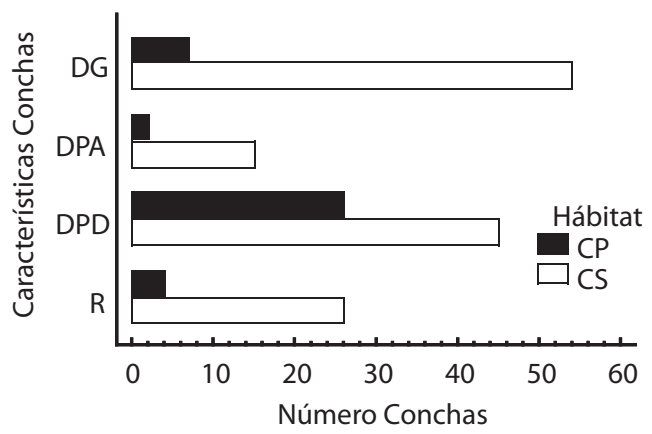

Fig. 3. Abundancia de conchas vacías dependiendo de las características presentes en cada hábitat (CP: cauce principal; CS; cauce secundario). DG: desgastada; DPA: depredada por ave; DPD: depredada por decápodo; R: rota.

Fig. 3. Abundance of empty shells relative to several characteristics and habitats (CP: main reach; CS: side reach). DG: eroded; DPA: preyed by birds; DPD: preyed by decapods; R: broken.

dos fuentes. Como se mostró anteriormente, las conchas depredadas por aves son más grandes que las depredadas por decápodos, sin embargo, ninguno de los dos tipos de daño presentó diferencias significativas entre hábitats (Fig. 4). El tamaño de las conchas desgastadas y rotas fue levemente diferente entre hábitats.

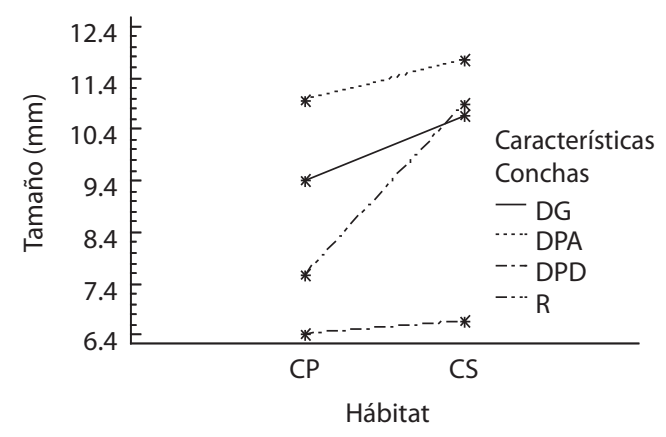

Fig. 4. Comparación del tamaño promedio entre las características de las conchas vacías y entre hábitats (CP: cauce principal; CS: cauce secundario). DG: desgastada; DPA: depredada por ave; DPD: depredada por decápodo; R: rota.

Fig. 4. Comparison of mean sizes among characteristics of empty shells, and between habitats (CP: main reach; CS: side reach). DG: eroded; DPA: preyed by birds; DPD: preyed by decapods; R: broken.

Los peces más comúnmente observados fueron los herbívoros de la familia Gobiidae (Cuadro 4). Mientras que los depredadores de las familias Eleotridae, Anguilidae y Mugilidae fueron poco frecuentes. No se observó a ningún pez alimentándose de $N$. virginea. No se observaron camarones grandes del género

CUADRO 3

Análisis de varianza de dos vías comparando el tamaño de las conchas entre dos hábitats (cauce principal y cauce secundario) y las características de las conchas (desgastadas, rotas y depredadas por aves o por decápodos), con los datos sin transformar logaritmicamente

TABLE 3

Two-Way analysis of variance comparing the size of shells between habitats (main reach and side reach) and shell characteristics (eroded, broken and preyed by birds or decapods) using untransformed data

$\begin{array}{lccccc}\text { FV } & \text { SC } & \text { GL } & \text { CM } & \text { VF } & \text { Pb } \\ \text { Hábitat } & 28.87 & 1 & 28.87 & 3.80 & 0.0529 \\ \text { Características Conchas } & 343.24 & 3 & 114.41 & 15.06 & <0.0001 \\ \text { Interacciones } & 28.42 & 3 & 9.47 & 1.25 & 0.29 \\ \text { Residuos } & 1299.45 & 171 & 7.60 & & \\ \text { Total Corregido } & 2090.65 & 178 & & & \end{array}$

*FV: Fuente de variación; SC: Suma de cuadrados; GL: Grados de libertad; CM: Cuadrados Medios; VF: Valor F; Pb: Probabilidad.

*FV: Source of variation; SC: Sum of squares; GL: Degrees of freedom; CM: Mean Square; VF: F-Ratio; Pb: Probability. 
CUADRO 4

Frecuencia relativa (porcentaje de fechas de muestreo) de peces observados en los censos subacuáticos en dos cauces del Río Mameyes, Puerto Rico

TABLE 4

Relative frequency (percent of sampling dates) of fishes in two reaches at Rio Mameyes, Puerto Rico

$\begin{array}{llcc}\text { Familia } & \text { Especie } & \text { CP* } & \text { CS* } \\ \text { Gobiidae } & \text { Awous tajasica } & 20 & 100 \\ & \text { Sicydium plumieri } & 20 & 100 \\ \text { Eleotridae } & \text { Eleotris pisonis } & 20 & 30 \\ \text { Anguilidae } & \text { Gobiomorus dormitor } & 0 & 20 \\ \text { Mugilidae } & \text { Anguilla rostrata } & 80 & 0 \\ *(\mathrm{CP}: \text { principal; main; } \text { CS: secundario; side). } & 30 & 40\end{array}$

Macrobrachium, aunque se observaron postlarvas migrando aguas arriba y algunos juveniles bajo las rocas.

\section{DISCUSIÓN}

Los resultados de este trabajo apoyan la hipótesis de Schneider \& Lyons (1993) que plantea que el alto riesgo de depredación que los juveniles de gasterópodos nerítidos experimentan en los estuarios y partes bajas de los ríos promueve su migración río arriba. En primer lugar, este trabajo establece que la depredación por aves y crustáceos en la parte baja del río Mameyes es menor que 2.5\%, el cual es mucho menor que la encontrada en ecosistemas costeros como los arrecifes y playas rocosas $(20-70 \%)$ en los cuales hay una mayor riqueza de depredadores especializados en moluscos (e.g. cangrejos) (Vermeij 1979). Vermeij (1993) sugiere que dado este bajo porcentaje de depredación en sistemas lóticos, sus gasterópodos presentan poca ornamentación, y que la baja depredación es la posible explicación para que algunos grupos de gasterópodos hayan invadido las aguas continentales a partir de linajes marinos.

Sin embargo, el valor observado por nosotros puede ser una subestimación debido a que peces depredadores engullen las conchas y no dejan rastro de depredación. Es sabido que en el estuario de los ríos de la zona se presentan varias especies de peces que incluyen a Neritina virginea como una parte importante de su dieta (Corujo-Flórez 1980). Mas aún algunos peces eleótridos como Gobiomorus dormitor y Eleotris picta, especializados en depredar gasterópodos nerítidos, pueden encontrarse varios kilómetros aguas arriba de los estuarios, aunque en bajas densidades como se observó en este trabajo. Por esta razón, sería interesante analizar la dieta de estos peces para tener una idea más completa acerca del impacto de la depredación sobre las poblaciones de gasterópodos. Sin embargo, es de anotar que peces insectívoros (Anguilla rostrata y Agonostomus monticola) y herbívoros (Awaous tajasica y Sicydium plumieri) fueron más abundantes y frecuentes que los moluscívoros (Eleotridae).

De acuerdo a nuestros resultados, los decápodos parecen ser los depredadores más importantes. De hecho, varias veces las conchas vacías fueron encontradas en grupos, lo cual sugiere una actividad oportunista del depredador sobre los juveniles migrantes. Contrario a los peces depredadores, los camarones Macrobrachium spp. son abundantes a lo largo de todo el río y su tamaño se incrementa aguas arriba (J. F. Blanco observaciones personales). 
Posiblemente, este tipo de depredadores utiliza tamaños predominantemente pequeños que coinciden con los de los individuos migrantes. El tamaño pequeño de los gasterópodos con perforaciones laterales sugiere la actividad de decápodos pequeños como los camarones y no de cangrejos cuyas quelas son significativamente más grandes. Adicionalmente, algunas de las conchas desgastadas que presentaban marcas de depredación por decápodos fueron más grandes que las intactas, lo cual podría sugerir que el desgaste disminuye el costo de manipulación de conchas más grandes lo cual está acorde con la teoría del forrajeo óptimo (Alcock 1998). Finalmente, debido a la amplia distribución de los camarones a lo largo del río y a que son depredadores acuáticos parece que la migración continua río arriba es una estrategia evolutivamente estable para los gasterópodos. Finalmente, se sugiere el uso de métodos diferentes a los censos visuales para estudiar los camarones Macrobrachium ya que aunque fueron poco frecuentes en nuestro estudio, se observaron muchas conchas depredadas por ellos. Adicionalmente, Johnson \& Covich (2000) encontraron que los camarones son más activos durante la noche y, por ello, otros métodos como la electropesca y las trampas podrían ser más recomendables, principalmente para capturar los individuos más grandes. Faenas recientes de electropesca realizadas en rápidos del área de estudio, mostraron que los camarones Macrobrachium carcinum representaron $0-6 \%$ de las capturas de individuos por unidad de esfuerzo, mientras que los peces eleótridos representaron el 6-31\%, Agonostomus monticola representó 6-10\% y Anguilla rostrata representó 38-47\% (J. F. Blanco, observaciones personales). En dichas faenas no se capturaron cangrejos E. sinuatifrons. Estos resultados apoyan la hipótesis de la baja abundancia de depredadores de moluscos en esta parte del río.

La depredación por aves, aunque está presente, es aparentemente baja $(<0.4 \%)$. El ave más común en el área fue la Gallinula chloropus la cual tiene una dieta especializada en insectos y gasterópodos y deja las conchas vacías en el lecho del río. Sin embargo, su densidad poblacional no parece ser lo suficientemente alta como para causar una presión significativa sobre la población de $N$. virginea. Otros depredadores potenciales son algunas garzas, aunque éstas generalmente llevan las conchas a sus perchas dejando acumulaciones fuera del río. Estas acumulaciones o concheros no fueron observadas en los recorridos por las orillas del tramo estudiado, pero Ford (1979) menciona que observó concheros a lo largo de quebradas en Hawaii. La depredación por aves de todas formas parece más enfocada en los gasterópodos más grandes y por lo tanto no explica la migración río arriba de juveniles. Además, los juveniles evitan las orillas y prefieren los rápidos (Blanco \& Scatena 2006) lo cual podría conferirles protección ante las aves depredadoras.

Independientemente del hábitat, la depredación por parte de decápodos parece ser más frecuente que por parte de aves. Sin embargo, la importancia relativa de una u otra parece influenciada por las características del hábitat. En los hábitats someros y de aguas que fluyen a baja velocidad, la proporción de conchas depredadas por aves es mayor que en los hábitats de mayor velocidad de flujo. Nuestros datos sugieren que hay una menor depredación tanto por aves como por decápodos en los rápidos, lo cual apoya la hipótesis planteada por Blanco \& Scatena $(2005,2007)$ en la cual la preferencia de hábitats y microhábitats profundos $\mathrm{y}$ de flujo rápido por parte de juveniles migrantes se debe al bajo riesgo de depredación. La ausencia de diferencias significativas de los tamaños de concha depredados por aves y por decápodos en los dos hábitats estudiados, indica la alta selectividad de los depredadores y que las tallas grandes y pequeñas, respectivamente, siguen siendo igual de vulnerables ante los depredadores.

El desgaste fue predominante a lo largo de todo el ámbito de tallas, lo cual está de acuerdo a la hipótesis de la suplementación de carbonato de calcio (Ferguson 1959). De esta forma aún los individuos más pequeños también experimentan la escasez del mismo tan pronto como abandonan los estuarios. $\mathrm{La}$ 
reabsorción de la espira y de las partes internas de la concha parece ser una respuesta a dichas limitaciones (Vermeij 1970), pero de todas formas, el desgaste puede ser responsable por pérdidas del peso de la concha de hasta $50 \%$ en los individuos más grandes (J. F. Blanco observaciones personales). Este desgaste significativo también parece ser un factor importante que determina el riesgo para que un gasterópodo sea depredado ya que debilita la concha y podría afectar el balance entre el gasto de manipulación y la ganancia energética por parte del depredador. Como se mencionó arriba las conchas desgastadas más grandes podrían ser utilizadas por los decápodos, ya que el tiempo de manipulación disminuiría y la ganancia energética sería mayor. Por su parte, las conchas desgastadas más pequeñas también podrían ser utilizadas por aves ya que aunque la ganancia energética sería baja el tiempo de manipulación sería menor.

Aunque mortalidad por depredación no parece ser un control importante de la dinámica poblacional de $N$. virginea, tiene el potencial de generar respuestas comportamentales en las tallas de individuos más susceptibles. Vermeij (1982) menciona que si la depredación fuese $100 \%$ exitosa no podría ser una fuerza evolutiva. De hecho, los intentos fallidos sobre los individuos y una incidencia mucho menor son frecuentes entre los depredadores de gasterópodos, particularmente entre los cangrejos. Por ello, la depredación por crustáceos en las partes bajas de los ríos podría ser una fuerza evolutiva importante que produce respuestas adaptativas como la migración río arriba de las tallas más vulnerables (Blanco \& Scatena 2005), la selección de microhábitats como las partes más profundas de los charcos y los rápidos (Blanco \& Scatena 2006), y la utilización de alarmas químicas (Schneider \& Frost 1986) observadas en los gasterópodos nerítidos.

Dado que cerca del $60 \%$ de las conchas vacías no presentaron signos de depredación se infiere que otras causas de mortalidad tales como catástrofes, enfermedades producto del debilitamiento de la concha y competencia intraespecífica también podrían ser importantes.
Por ejemplo, las conchas rotas, en su mayoría de tallas intermedias que se encuentran expuestas sobre las rocas, pueden ser el producto de aplastamiento durante las crecientes, mientras que las conchas desgastadas y vacías que presentaron varias perforaciones hechas con rádulas que atravesaban la voluta del cuerpo, podrían reducir la eficacia biológica de sus habitantes (Vermeij 1993). Finalmente, algunas conchas vacías $(2.5 \%)$, principalmente pequeñas, fueron encontradas sin rastros de daños o marcas de depredación, lo cual ha sido sugerido por Blanco (2005) como mortalidad por inanición producto de una asimetría competitiva entre gasterópodos juveniles y adultos.

Se sugiere que estudios posteriores exploren: 1) la contribución a la depredación por parte de peces, particularmente eleótridos, 2) la variación de la incidencia de depredación a lo largo del río por parte de diferentes depredadores y 3) las respuestas comportamentales de los gasterópodos ante los diferentes depredadores.

\section{AGRADECIMIENTOS}

Esta investigación fue financiada por el Acuerdo de Cooperación 00-CA-11120101-004 entre el Instituto Internacional de Dasonomía Tropical del Servicio Forestal de los Estados Unidos y la Universidad de Puerto Rico, Río Piedras Campus. Financiamiento adicional fue proporcionado por los proyectos "Programa de Investigación Ecológica a Largo Plazo en el Bosque Experimental de Luquillo" y "Modeling complex interactions of overlapping river and road networks in a changing landscape" financiados por la Fundación Nacional para las Ciencias. Se agradece a Eilee Tejada, Norma Santiago, Ivette Ruiz, Saúl Rodríguez, Vyrmar Mangual, Andrés Fernández y Sara Raquel López por su ayuda voluntaria en el campo y el laboratorio. El Laboratorio de Limnología Tropical de la Universidad de Puerto Rico, Río Piedras dirigido por Alonso Ramírez y Jorge Ortiz proveyó espacio físico. Contribución LIMNOBASE-ELICE No. 2. 


\section{RESUMEN}

Se compararon las abundancias y tamaños de conchas del gasterópodo Neritina virginea de acuerdo a los daños presentados en especimenes vivos y muertos $(n=5066)$ recolectados en el río Mameyes, Puerto Rico (Antillas Mayores). Se observaron daños por depredación de aves y camarones, y signos de erosión por la acción del raspado de la rádula por parte de otros individuos de la misma especie. La depredación fue aparentemente baja $(<2.5 \%)$, pero los tamaños de concha depredados por aves y camarones fueron significativamente diferentes. Las primeras se especializan en los individuos residentes más grandes, mientras que los segundos se especializan en individuos juveniles que presentan un comportamiento migratorio. Estos tamaños no fueron diferentes entre dos hábitats, aunque se observó más conchas depredadas por decápodos que por aves. El desgaste fue común en conchas de todos los tamaños. A pesar de su baja contribución a la dinámica poblacional de los gasterópodos, la depredación podría ser un control importante de respuestas de comportamiento dependientes del tamaño, tales como la migración río arriba y la selección de microhábitats.

Palabras claves: Neritina virginea, gasterópodos migratorios, efectos dependientes de la talla, daños de conchas, depredación

\section{REFERENCIAS}

Alcock, J. 1998. Animal behavior. Sinauer. Massachusetts, EEUU.

Alpírez, O., J.M. Ureña \& R. Brenes. 1984. Hallazgo de un depredador de Neritina latissina (Gastropoda: Neritidae) en el río Punta Mala, Costa Rica. Rev. Biol. Trop. 32: 159-160.

Blanco, J.F. 2005. Physical habitat, disturbances, and the population ecology of the migratory snail Neritina virginea (Gastropoda: Neritidae) in streams of Puerto Rico. Tesis Doctoral, Departamento de Biología, Universidad de Puerto Rico, Recinto de Río Piedras, Puerto Rico.

Blanco, J.F. \& F.N. Scatena. 2005. Floods, habitat hydraulics and upstream migration of Neritina virginea (Gastropoda: Neritidae) in Northeastern Puerto Rico. Carib. J. Sci. 41:55-74.

Blanco, J.F. \& F.N. Scatena. 2006. Hierarchical contribution of river-ocean connectivity, water chemistry, hydraulics, and substrate to the distribution of diadromous snails in Puerto Rican streams. J. N. Am. Benthol. Soc. 25: 82-98.
Blanco, J.F. \& F.N. Scatena. 2007. The spatial arrangement of Neritina virginea (Gastropoda: Neritidae) during upstream migration in a split-channel reach. River Res. Appl. 23: 235-245.

Corujo-Flores, I.N. 1980. A study of fish populations in Espíritu Santo River estuary. Tesis de Maestría, Departamento de Biología, Universidad de Puerto Rico, Recinto de Río Piedras, Puerto Rico.

Crandall, E.D. 1999. Early life history aspects of amphidromous neritid snails in Moorea, French Polynesia. Berkeley Sci. 3: 98-102.

García-Cubas, A. 1981. Moluscos de un sistema lagunar tropical en el sur del Golfo de México (Laguna de Términos, Campeche). Inst. Cienc. Mar Limnol, UNAM, Publ. Esp. 5: 1-182.

Ferguson, F.F. 1959. Intraspecific predation in a Puerto Rican neritid snail. Trans. Amer. Microsc. Soc. 78: 240-242.

Ford, I.J. 1979. Biology of a Hawaiian fluvial gastropod Neritina granosa Sowerby (Prosobranchia: Neritidae). Tesis de Maestría, Universidad de Hawaii, Honolulu, Hawaii.

Johnson S.L. \& A.P. Covich. 2000. Day and night differences in freshwater shrimp foraging activity as related to instream flow. Reg. Rivers 16: 91-99.

Lampert, W. \& U. Sommer. 1997. Limnoecology. The ecology of lakes and streams. Editorial de la Universidad de Oxford, Nueva York, EEUU.

Layman, A.C. \& B.R. Silliman. 2002. Preliminary survey and diet analysis of juvenile fishes of an estuarine creek on Andros Island, Bahamas. Bull. Mar. Sci. 70: 199-210.

Pyron, M. \& A. P. Covich. 2003. Migration patterns, densities and growth of Neritina punctulata snails in Río Espíritu Santo and Río Mameyes, Northeastern Puerto Rico. Carib. J. Sci. 39: 338-347.

Resh, V.H., M. Moser \& M. Poole. 1999. Feeding habits of some freshwater fishes in streams of Moorea, French Polynesia. Ann. Limnol. 35: 205-210.

Schneider, D.W. \& T.M. Frost. 1986. Massive upstream migrations by a tropical freshwater neritid snail. Hydrobiologia 137: 153-157.

Schneider, D.W. \& J. Lyons. 1993. Dynamics of upstream migration in two species of tropical freshwater snails. J. North Amer. Benthol. Soc. 12: 3-16. 
Texeira, L. R. 1994. Abundance, reproductive period, and feeding habits of eleotrid fishes in estuarine habitats of north-east Brazil. J. Fish Biol. 45: 749-761.

Vermeij, G. 1970. Adaptive versatility and skeleton construction. Am. Nat. 104: 253-260.

Vermeij, G. 1979. Shell architecture and causes of death of micronesian reef snails. Evolution 33: 686-696.
Vermeij, G. 1982. Unsuccessful predation and evolution. Am. Nat. 120: 701-720.

Vermeij, G. 1993. A natural history of shells. Editorial de la Universidad de Princeton, Nueva Jersey, EEUU.

Warmke, L.G. \& D.S. Erdman. 1963. Records of marine molluscs eaten by bonefish in Puerto Rican waters. Nautilus 76: 115-120. 